Pacific Journal of Mathematics

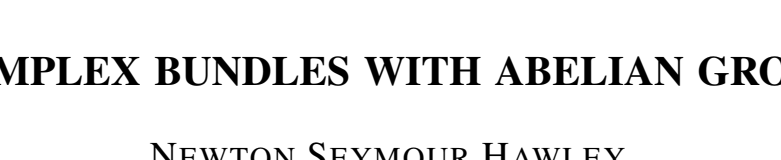




\section{COMPLEX BUNDLES WITH ABELIAN GROUP}

\section{N. S. HAWLEY}

We shall be concerned here with certain complex bundles whose bundle groups are Abelian and with some bundle spaces of such bundles, when a particular fibre is employed. We begin with a general definition of complex bundle and attempt to describe some of the work of Kodaira and Spencer in this context. A general theory is then described. This is followed by considering a special case, the group being that of a one dimensional Abelian variety, that is, the additive group of complex numbers reduced modulo a discrete subgroup which is generated by two complex numbers whose ratio is not real. Certain efforts toward an explicit classfication of such bundles are made, together with an attempt to relate the concepts here introduced to some in the Kodaira-Spencer theory, and to some more classical notions. A version of an Abel's theorem is given.

The last section of the paper is related to the "general theory", mentioned above, only to the extent that the spaces considered are the bundle spaces of bundles with an Abelian group. Most of the results and statements in the last section are not dependent on the earlier sections of the paper, and are perhaps related to the earlier sections only in the author's mind, because they arose at the same time and from the same considerations. The principal result there is a construction, involving Hopf manifolds, which shows that Kählerian structure is very rare among compact complex manifolds.

1. Complex bundles and the Kodaira-Spencer theory. Let $V$ be a complex manifold, $\mathscr{U}=\left\{U_{i}\right\}$ an indexed covering of $V$ by coordinate neighborhoods, $N(\mathscr{U})$ the nerve of the covering $\mathscr{U}, G$ a complex Lie group, ( 5 the sheaf of germs of complex analytic mappings of $V$ into $G$. Suppose analytic mappings $\theta_{i j}: U_{i} \cap U_{j} \rightarrow G$ are given, such that

$$
\begin{array}{cr}
\theta_{i j}(x) \cdot \theta_{j k}(x) \cdot \theta_{k i}(x)=\text { identity, } & \text { for } x \in U_{i} \cap U_{j} \cap U_{k}, \\
\theta_{i i}(x)=\text { identity, } & \text { for } x \in U_{i} .
\end{array}
$$

We say the set $\left\{\theta_{i j}\right\}$ defines a $\mathscr{\ell}$-coordinate bundle with respect to $G$, or merely a $\mathscr{Z}$-bundle. The $\theta_{i j}$ are called the coordinate transformations. Two $\mathscr{C}$-bundles $\left\{\theta_{i j}\right\}$ and $\left\{\theta_{i j}^{\prime}\right\}$ are said to be $\mathscr{\ell}$-equivalent (with respect to $G$ ) if there exist analytic mappings $\lambda_{i}: U_{i} \rightarrow G$, such that

$$
\theta_{i j}^{\prime}=\lambda_{i} \theta_{i j} \lambda_{j}^{-1}
$$

for $x$ in $U_{i} \cap U_{j}$.

Received December 22, 1954. 
Let $\mathscr{T}(V, G ; \mathscr{U})$ be the set of equivalence classes of $\mathscr{U}$-bundles over $V$. If $\mathscr{U}^{\prime}$ is a refinement of $\mathscr{U}$, we have natural simplicial maps $\mu: N\left(\mathscr{U}^{\prime}\right) \rightarrow N\left(\mathscr{Q}^{\ell}\right)$, which give rise to definite restriction maps $\mu^{*}$ of the $\mathscr{C}$-bundles into the $\mathscr{U}^{\prime}$-bundles, $\left(\mu^{*} \theta\right)_{r s}=\theta_{\mu(r) \mu(s)}$ in $U_{r}^{\prime} \cap U_{s}^{\prime}\left({ }_{1}{ }_{1} U_{r}^{\prime}\right\}=$ $\left.\mathscr{U}^{\prime}\right)$, thus defining a mapping $\hat{\mu}: \mathscr{F}\left(V, G ; \mathscr{U}^{\prime}\right) \rightarrow \mathscr{T}\left(V, G ; \mathscr{U}^{\prime}\right)$ which is independent of the particular $\mu$ chosen. Define $\mathscr{F}(V, G)$ to be the direct limit of the $\mathscr{F}(V, G ; \mathscr{Q})$. The elements of $\mathscr{F}(V, G)$ are the $G$-bundles over $V .^{1}$

If we impose the restriction that $G$ shall be Abelian then $\mathscr{F}(V, G)$ becomes a group. For if $B$ is a $G$-bundle defined by $\left\{\theta_{i j}\right\}$ and if $B^{\prime}$ is defined by $\left\{\theta_{i j}^{\prime}\right\}$ then $B^{\prime \prime}$, the $G$-bundle defined by $\theta_{i j}^{\prime \prime}=\theta_{i j} \theta_{i j}^{\prime}$ (writing the group operation in $G$ multiplicatively) is the "sum" of $B$ and $B^{\prime}$, $B^{\prime \prime}=B+B^{\prime}$. (The reason for choosing the multiplicative notation in one case and the additive in the other will become clear later.)

From the definitions of $\mathscr{F}(V, G)$ and $H^{1}(V,(S)$ it is clear that we may identify the two, so we write $\mathscr{F}(V, G)=H^{1}(V,(s)$. (See [14] for definitions of $H^{p}(V, S)$ where $S$ is any sheaf.)

Kodaira and Spencer have developed a theory of multiplicative line bundles [10]. In order to proceed by analogy let us first reformulate the elements of their theory.

Consider the exact sequence of groups,

$$
0 \rightarrow Z \stackrel{i}{\longrightarrow} C \stackrel{e}{\longrightarrow} C^{*} \rightarrow 0,
$$

where $Z$ is the additive group of integers, $C$ is the additive group of complex numbers, and $C^{*}$ is the multiplicative group of non-zero complex numbers. The isomorphism of $Z$ into $C$ is defined by inclusion; the homomorphism of $C$ onto $C^{*}$ is defined by the exponential operator $e[c]=\exp c=e^{2 \pi i c}$. We have the corresponding exact sequence of sheaves over our manifold $V$,

$$
0 \rightarrow \boldsymbol{Z} \rightarrow \Omega \rightarrow \Omega^{*} \rightarrow 0 .
$$

$\Omega$ is the sheaf of germs of holomorphic functions on $V$, and $\Omega^{*}$ is the sheaf of germs of non-vanishing holomorphic functions on $V ; Z$ is merely the sheaf $V \times Z$.

Corresponding to (2) we have the exact cohomology sequence

$$
0 \rightarrow H^{0}(V, \boldsymbol{Z}) \rightarrow H^{\nu}(V, \Omega) \rightarrow H^{0}\left(V, \Omega^{*}\right) \rightarrow H^{1}(V, \boldsymbol{Z}) \rightarrow \cdots .
$$

We observe that $H^{0}(V, Z) \approx Z, H^{0}(V, \Omega) \approx C, H^{0}\left(V, \Omega^{*}\right) \approx C^{*}, H^{q}(V, Z) \approx$ $H^{q}(V, Z)$. Furthermore we know that $H^{q}(V, \Omega)=H^{0, q}(V)$ and $H^{1}\left(V, \Omega^{*}\right)=$ $\mathscr{F}\left(V, C^{*}\right)$, the group of multiplicative line bundles over $V$. With this

1 This approach to defining $G$-bundles is due to M. F. Atiyah.

2 The manifolds in question are assumed to be compact. While many of the definitions are applicable in general, most of the assertions will hold only if we assume compactness. 
information, we can derive, from the sequence above, the exact sequence

$$
0 \rightarrow Z \rightarrow C \rightarrow C^{*} \rightarrow H^{1}(V, Z) \rightarrow H^{0,1}(V) \rightarrow \mathscr{F}\left(V, C^{*}\right) \rightarrow \cdots
$$

Denoting $\mathscr{F}\left(V, C^{*}\right)$ by $\mathscr{F}$, and using (1) on the first three terms of (3) we have

$$
0 \rightarrow H^{1}(V, Z) \stackrel{n}{\longrightarrow} H^{0,1}(V) \stackrel{\alpha}{\longrightarrow} \mathscr{F} \stackrel{c}{\longrightarrow} H^{2}(V, Z) \rightarrow \cdots .
$$

In case the manifold $V$ is Kähler, the homomorphisms $h, \alpha, c$ all have interpretations which are significant independent of this formalism. The homomorphism $h$ is simply the operator $\Pi_{0,1} H$, where $H$ is Hodge's operator and $\mathrm{II}_{0,1}$ is a purification ${ }^{3}$ operator [4]. The homomorphism $c$ merely assigns to each $C^{*}$-bundle $" \in \mathscr{F}$, its Chern class $c(") \in H^{2}(V, Z)$. The operator $\alpha$ is a little more difficult to describe. Let $\dddot{Z}$ be a covering of $V$, let $(i)$ be a point in $U_{i} \in \%$, let $\bar{\varphi}$ be a $(0,1)$ form on $V$. Define $\theta_{i j}=\exp \left\{\int_{(i)}^{(j)} \bar{\varphi}\right\}$ when $U_{i} \cap U_{j} \neq 0$. The $\theta_{i j}$ define a $\mathscr{U}$-bundle. Let $l$ be its image (under the direct limit) in $\mathscr{F}$. Then $\alpha$ maps $\bar{\varphi}$ onto $\measuredangle$.

The kernel of the homomorphism $c$ is denoted by $\mathfrak{P}$; it is $\alpha\left(H^{0,1}(V)\right)$ and is isomorphic to $\left.H^{0,1}(V) / / H^{1}(V, Z)\right)$. \& is called, by Kodaira and Spencer, the Picard variety of $V$. In case $V$ is a Kähler manifold $\mathfrak{B}$ is a complex multitorus.

This material thus far is not particularly significant, it becomes so when the connection between $C^{*}$-bundles and divisors is revealed. A relationship is thus established between the bundle-sheaf theory and the classical theory of linear (and other) systems on algebraic varieties.

A divisor $D$ on $V$ determines a $C^{*}$-bundle in the following way. Let $\mathscr{U}$ be a covering of $V$ such that $D$ is defined in each coordinate neighborhood $U_{i}$ by a minimal local equation $R_{i}=0$. Define $\theta_{i j}$ by

$$
\theta_{i j}(x)=\frac{R_{i}(x)}{R_{j}(x)} \quad \text { for } \quad x \in U_{i} \cap U_{j} .
$$

The $\theta_{i j}$ determines a $\%$-bundle which, in turn, gives rise to a unique $C^{*}$-bundle, which we denote by $|D|$. Thus the divisors on $V$ correspond to 1-dimensional cocycles with coefficients in $\Omega^{*}$, and divisor classes (or complete linear systems) correspond to cohomology class, or elements of $H^{1}\left(V, \Omega^{*}\right)$.

The central result of the Kodaira-Spencer theory is that, for the case where $V$ is an algebraic variety, every $C^{*}$-bundle arises from a divisor class.

2. The general theory. Let $E$ denote a simply connected Abelian

3 This very descriptive term is due to E. Calabi. 
complex Lie group (not necessarily connected), and $D$ a discrete subgroup. Let $G$ be the factor group so that we have

$$
0 \rightarrow D \rightarrow E \rightarrow G \rightarrow 0 .
$$

Denote the group $\mathscr{F}(V, G)$ of $G$ bundles over $V$ by $\mathscr{Q}$, and let

$$
0 \rightarrow \Delta \rightarrow E \rightarrow \Gamma \rightarrow 0
$$

be the sequence of sheaves over $V$ arising from (5) above. The corresponding cohomology sequence is

$$
0 \rightarrow H^{0}(V, \Delta) \rightarrow H^{\nu}(V, E) \rightarrow H^{0}(V, \Gamma) \rightarrow H^{1}(V, \Delta) \rightarrow \cdots .
$$

By observing that $H^{\circ}(V, \Delta) \approx D, H^{\nu}(V, E) \approx E, H \cdot(V, \Gamma) \approx G$, and $H^{p}(V, \Delta)$ $\approx H^{p}(V, D)$, and by using (5) we may write

$$
0 \rightarrow H^{1}(V, D) \stackrel{\check{\prime}}{\longrightarrow} H^{1}(V, E) \stackrel{\tilde{a}}{\longrightarrow} \mathscr{S} \stackrel{\tilde{c}}{\longrightarrow} H^{2}(V, D) \rightarrow H^{2}(V, E) \rightarrow \cdots .
$$

If $\ell \in \mathscr{B}$ then $c(\ell)$ is again the Chern class of the bundle; $z$ and $\tilde{\alpha}$ could also be given direct interpretations, but this is not worthwhile in general since the general theory lacks significance and is enunciated only to gain perspective.

The kernel of $\tilde{c}$ is $\mathscr{F}=\tilde{\alpha}\left(H^{1}(V, E)\right)$, it is again the group of $G$-bundles which are topologically trivial.

3. Elliptic bundles. To obtain results of significance (that is, which can be interpreted geometrically on $V$ ) we must specialize $G$. The most significant specialization is $G=C^{*}$, the Kodaira-Spencer theory. Another specialization of some interest is $G=T_{\omega}$, where $T_{\omega}$ is the "elliptic" group with periods $\omega=\left(\omega_{1}, \omega_{2}\right)$, that is, $\omega_{1}$ and $\omega_{2}$ are complex numbers such that $\omega_{1} / \omega_{2}$ is not real, and $T_{\omega}$ is $C \bmod \left(\omega_{1}, \omega_{2}\right)$.

Let $\Lambda_{\omega}$ be the "lattice" subgroup of $C$ which is generated by $\omega_{1}$ and $\omega_{2}$. We have the exact sequence

$$
0 \rightarrow \Lambda_{\omega} \stackrel{i}{\longrightarrow} C \stackrel{\omega}{\longrightarrow} T_{\omega} \rightarrow 0,
$$

where the homomorphism $i$ is inclusion, and we denote by $\omega$ the homomorphism of $C$ onto $T_{\omega}$ arising from reduction $\bmod \left(\omega_{1}, \omega_{2}\right)$. Of course, the homomorphism $\omega$ of $C$ onto $T$ is not uniquely determined by the analytic structure of $T_{\omega}$; this analytic structure on $T$ determines $\left(\omega_{1}\right.$, $\left.\omega_{2}\right)$ only up to a modular substitution. For the following discussion we choose a fixed (but arbitrary) $\omega$ from among its equivalence class, under the modular group. We may also note that for the previous theory of multiplicative line bundles, the homomorphism $e: C \rightarrow C^{*}$ given by the exponential map is not unique and is saved from "arbitrariness" only by tradition. (We shall henceforth usually drop the subscript $\omega$ unless 
it becomes useful to discuss the different conformal structures on $T_{\omega}$.) We have the sequence of sheaves over $V$,

$$
0 \rightarrow \Lambda \rightarrow \Omega \rightarrow T \rightarrow 0 .
$$

This gives the cohomology sequence

$$
0 \rightarrow H^{0}(V, \boldsymbol{\Lambda}) \rightarrow H^{0}(V, \Omega) \rightarrow H^{:}(V, \boldsymbol{T}) \rightarrow H^{1}(V, \Lambda) \rightarrow \cdots .
$$

Again we observe that $H^{0}(V, \boldsymbol{d}) \approx A, H^{0}(V, \Omega) \approx C, H^{\circ}(V, \boldsymbol{T}) \approx T, H^{\nu}(V, \boldsymbol{A})$ $\approx H^{p}(V, \Lambda), \quad H^{p}(V, \Omega) \approx H^{0, p}(V), \quad H^{1}(V, T)=\mathscr{B}=\mathscr{F}(V, T)$. So, as before, we get an exact sequence

$$
0 \rightarrow H^{1}(V, \Lambda) \stackrel{\hat{\iota}}{\longrightarrow} H^{0,1}(V) \stackrel{\hat{x}}{\longrightarrow} \mathscr{S} \stackrel{\hat{c}}{\longrightarrow} H^{2}(V, \Lambda) \rightarrow H^{0,2}(V) \rightarrow \cdots
$$

by using (8), (9), and the isomorphisms we just observed.

The homomorphism $\hat{\alpha}$ is defined in the same way as $\alpha$, when $V$ is Kählerian, except we now define $\theta_{i j}=\omega\left\{\int_{(i)}^{(j)} \bar{\varphi}\right\}$, that is, we use the homomorphism $\omega$ instead of $e=\exp$, thus giving rise to an element of $\mathscr{B}$ instead of $\mathscr{F}$. Since there is a natural mapping $H^{1}(V, A) \rightarrow H^{1}(V, C)$, we can again realize $\hat{k}$ as the Hodge operator followed by a purification operator. For $a \in \mathscr{S}, \hat{c}(\measuredangle)$ is the Chern class of $a$; it is an element of $H^{2}(V, \Lambda)$, since $\pi_{1}(T)$, the "fundamental group" of $T$, is actually $A$.

From the homomorphism $C \stackrel{e}{\longrightarrow} C^{*}$ and the homomorphism $C \stackrel{\omega}{\longrightarrow} T$, we can define a homomorphism $C^{*} \stackrel{\sigma}{\longrightarrow} T$ such that $\omega=\sigma e .^{4}$ The mapping $\sigma$ induces a natural homomorphism of $\mathscr{F}$ into $\mathscr{S}$, consequently we may state: Every $C^{*}$-bundle gives rise to a unique T-bundle (for each $T$ ). Of course, many different $C^{*}$-bundles may give rise to the same $T$-bundle.

In particular we see that every divisor gives rise to a T-bundle, thus allowing us to introduce a new kind of equivalence among divisors on $V$, elliptic equivalence, or more precisely $\omega$-equivalence, since we get a different equivalence for each $T$.

The general question naturally arises as to whether every $T$-bundle arises from a $C^{*}$-bundle: and, more particularly, when $V$ is algebraic, does every $T$-bundle arise from a divisor? The answer to these questions is the negative. In order to see this we construct a new sequence.

Let $\Delta$ be the kernel of $\sigma$, so that $C^{*} \mid \Delta=T$, and let $\rho$ be an isomorphism of $Z$ onto $4 .^{5}$

Then we have

$$
0 \rightarrow Z \stackrel{\rho}{\longrightarrow} C^{*} \stackrel{\sigma}{\longrightarrow} T \rightarrow 0
$$

${ }^{4}$ Of course, the $\sigma$ we define is not unique. However, we pick such a $\sigma$ for each $\omega$, and these $\sigma$ 's remain fixed for our entire discussion. Whenever any assertion of uniqueness is made we must consider it in the light of this somewhat free choice of $\sigma$.

5 We should remark that, like $\omega$ and $\sigma$, the isomorphism $\rho$ is not unique. 
In fact we can write

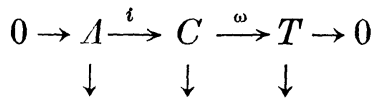

$$
\begin{aligned}
& 0 \rightarrow Z \stackrel{\rho}{\longrightarrow} C^{*} \stackrel{\sigma}{\longrightarrow} T \rightarrow 0 .
\end{aligned}
$$

From these we can deduce the exact sequences with associated homomorphisms

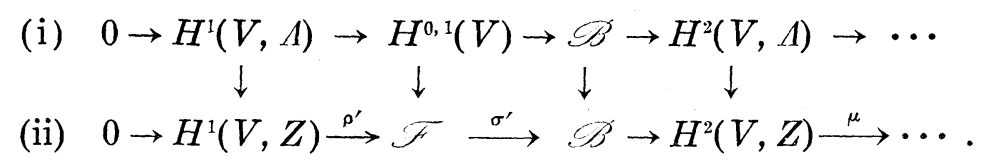

First, to draw a few conclusions from this, suppose $H^{1}(V, Z)=$ $H^{2}(V, Z)=0$. Then, from the sequences $(12)$, we have $\mathscr{F} \approx \mathscr{S} \approx H^{0,1}(V)$, that is to say that the groups of $C^{*}$-bundles, $C$-bundles, and $T$-bundles (for every $T$ ) are all isomorphic to each other. If $H^{1}(V, Z)=0$, then $\mathscr{F}$ is isomorphic to a subgroup of $\mathscr{B}$. Conversely if $\mathscr{F}$ is isomorphic to a subgroup of $\mathscr{S}$ then $H^{1}(V, Z)=0$. From $(12$,ii) we see that if the kernel of $\mu$ is not zero then there exist $T$-bundles which do not arise from any $C^{k}$-bundle. As we shall see, this is often the case even for algebraic varieties.

Let $g_{p}=\operatorname{dim} H^{p, 0}(V), \bar{g}_{p}=\operatorname{dim} H^{0, p}(V)$. When $V$ is a Kähler manifold (so in particular when it is algebraic) we have $g_{p}=\bar{g}_{p}$. Furthermore we denote the $p$ th Betti number of $V$ by $R_{p}$.

Theorem I. If $R_{2}>R_{3}+2 \bar{g}_{2}$ on $V$ then there exist T-bundles which do not arise from $C^{*}$-bundles on $V$.

Proof. We shall need the exact sequences

$(\alpha) \quad \cdots \rightarrow \mathscr{F} \rightarrow H^{2}(V, Z) \rightarrow H^{0,2}(V) \rightarrow H^{2}\left(V, \Omega^{*}\right) \rightarrow H^{3}(V, Z) \rightarrow \cdots$

(ß) $0 \rightarrow H^{1}(V, Z) \rightarrow \mathscr{F} \rightarrow \mathscr{S} \rightarrow H^{2}(V, Z) \stackrel{\mu}{\longrightarrow} H^{2}\left(V, \Omega^{*}\right) \rightarrow \cdots \cdot$

We must show that the kernel of $\mu$ is not zero. Denote the component of the identity of $H^{2}\left(V, \Omega^{*}\right)$ by $I^{2}\left(V, \Omega^{*}\right)$. Let $k$ denote the rank of $H^{2}\left(V, \Omega^{*}\right) / I^{2}\left(V, \Omega^{*}\right)$, and let $q$ denote the rank of that part of the image of $H^{2}(V, Z)$ in $H^{2}\left(V, \Omega^{*}\right)$ which lies in $I^{2}\left(V, \Omega^{*}\right)$. From $(\alpha)$ We see that $I^{2}\left(V, \Omega^{*}\right)$ is a complex vector space, of $\operatorname{dim} \bar{g}_{2}$, reduced modulo a discrete subgroup. The rank of this subgroup must be $\leqq 2 \bar{g}_{2}$. Also from $(\alpha)$ we see that $H^{2}\left(V, \Omega^{*}\right) / I^{2}\left(V, \Omega^{*}\right)$ is isomorphic to a subgroup of $H^{3}(V, Z)$, since $H^{0,2}(V)$ is connected. Thus $k \leqq R_{3}$ and $q \leqq 2 \bar{g}_{2}$. Therefore the image of $H^{2}(V, Z)$ in $H^{2}\left(V, \Omega^{*}\right)$ must be of $\operatorname{rank} \leqq R_{3}+2 \bar{g}_{2}<R_{2}$. But this means the kernel of $\mu$ is $\neq 0$. 
CoRollary 1. If $V$ is compact Kähler then $g_{2}=R_{3}=0$ is sufficient to insure the existence of $T$-bundles not arising from $C^{*}$-bundles.

For in this case $g_{2}=\bar{g}_{2}$ and $R_{2}>0$. Specific examples are furnished by the complex projective spaces and, more generally, by the rational homogeneous algebraic manifolds; if we restrict the manifold to be an algebraic surface, we can drop the condition of homogeneity. In fact, for an algebraic surface we can state:

COROLlaRY 2. If $V$ is an algebraic surface and if $I>2\left(p_{a}-1\right)$ then there exist $T$-bundles not arising from any divisor.

Here $p_{a}$ means the arithmetic genus of the surface $V$, and $I$ stands for the Zeuthen-Segre invariant, which is defined as follows: if $\{\mathscr{C}\}$ is an irreducible linear pencil of curves (on $V$ ), of genus $\pi$ and degree $n$, and if $\delta$ is the number of curves in $\{\mathscr{C}\}$ which are of genus $\pi-1$, then we define $I=\delta-n-4 \pi$, [18]. In order to derive the corollary we observe that, for an algebraic surface, $\bar{g}_{2}=g_{2}=p_{g}$ the geometric genus, and $R_{3}=R_{1}=2 q$, where $q=p_{q}-p_{a}$ is the irregularity of $V$. Furthermore it is known that $I+4$ is equal to the Euler characteristic of $V$. Hence $R_{2}=I+4 q+2$. By the theorem, $I+4 q+2>2 q+2 p_{g}$ or $I>2\left(p_{g}-q-1\right)=$ $2\left(p_{a}-1\right)$ is sufficient to imply the existence of the bundles asserted.

The task of classifying all $T$-bundles over $V$ is made relatively easy by the circumstance that $\mathscr{P}$ is the group $H^{1}(V, \boldsymbol{T})$. Let $\mathscr{E}$ denote the kernel of $\hat{c}$ in the exact sequence (10), and $\mathscr{D}$ denote the image in $H^{2}(V, A)$ of $B$ under $\hat{c}$. We then have the sequence

$$
0 \rightarrow \mathscr{E} \rightarrow \mathscr{S} \rightarrow \mathscr{X} \rightarrow 0 .
$$

Actually $\mathscr{E}$ is the component of the identity in $\mathscr{P}$, thus $\mathscr{D}$ is discrete. We may thus write

$$
\mathscr{D}=\mathscr{E} \times \mathscr{D} \text {. }
$$

In order for this calculation to be effective we must "know" $\mathscr{E}$ and $\mathscr{D}$. But we have at our disposal the information that

$$
\mathscr{E} \approx H^{0,1}(V) / \hat{\hbar}\left(H^{1}(V, A)\right) .
$$

Furthermore, when $V$ is an algebraic variety, $H^{0,1}(V)$ can be constructed effectively and explicitly-as the space of conjugates of the Picard differentials of the first kind. If $H^{p}(V)$ denotes the $p$-dimensional cohomology group of $p$-forms with complex coefficients

$$
H^{p}(V)=\sum_{r+s=p} \oplus H^{r, s}(V) \text {. }
$$


Let $H^{p}(V, C)$ denote the $p$-dimensional cohomology group with complex coefficients. There is a natural isomorphism

$$
n: H^{p}(V, C) \underset{\text { onto }}{\longrightarrow} H^{p}(V),
$$

there is also an inclusion map

$$
\iota: H^{p}(V, \Lambda) \rightarrow H^{p}(V, C) .
$$

Since $\mathscr{C} C H^{2}(V, A)$ goes into zero under the homomorphism of $H^{2}(V, A)$ into $H^{0,2}(V)$ defined in (10), we see that

$$
n_{\ell} \mathscr{C} \subset H^{2,0}(V) \oplus H^{1,1}(V) \text {. }
$$

In case $V$ is Kähler and $g_{2}=0$, we may assert that $n \iota \mathscr{D} \subset H^{1,1}(V)$, that is, each element is of type $(1,1)$.

Turning to the question of a geometric interpretation of the $\omega$ equivalence defined earlier, we are again thrown back on the sequence $(12, \mathrm{ii})$. Let $D^{\prime}$ and $D^{\prime \prime}$ be two divisors on $V$ and let $D=D^{\prime}-D^{\prime \prime}$. In order for $D^{\prime}$ to be $\omega$-equivalent to $D^{\prime \prime}$, or what is the same, for $D$ to be $\omega$-equivalent to zero, it is necessary for $D^{\prime}$ to be homologous to $D^{\prime \prime}$, or $D$ to be homologous to zero. (For if the $T$-bundle determined by $D^{\prime}-D^{\prime \prime}=D$ is $\omega$-trivial, it is certainly topologically trivial.) Thus let us suppose $D^{\prime}-D^{\prime \prime}=D \sim 0$.

There exists on $V$ a multiplicative meromorphic function having $D$ as its divisor [13]. Let $z$ be a variable point on $V$ and let $f(z)$ be a multiplicative meromorphic function having $D$ as its divisor. Let $r$ be a 1-cycle on $V$ and let us continue $f(z)$ around $\gamma$; then $f(z)$ goes into $\chi_{D}(\gamma) \cdot f(z)$, where $\chi_{D}(\gamma)$ is a complex number of modulus 1. Another way of getting at this number is

$$
\int_{\gamma} d \log f(z)=\log \chi_{D}(\gamma), \quad \text { or } \chi_{D}(\gamma)=\exp \left\{\int_{\gamma} d \log f(z)\right\} .
$$

From this expression it is easy to see that

$$
\chi_{D}\left(\gamma_{1}+\gamma_{2}\right)=\chi_{D}\left(\gamma_{1}\right) \cdot \chi_{D}\left(\gamma_{2}\right)
$$

Furthermore let $\gamma$ and $\gamma^{\prime}$ be two representatives of the same element of $H_{1}(V, Z)$, then

$$
\chi_{D}(\gamma)=\chi_{D}\left(\gamma^{\prime}\right)
$$

This last statement follows directly from (13). Finally, suppose $g(z)$ is another multiplicative meromorphic function having $D$ as its divisor, then $g(z) / f(z)=c$ would be everywhere finite (and single valued) on $V$, thus constant. Now, 


$$
\int_{\gamma} d \log g(z)=\int_{\gamma} d \log c f(z)=\int_{\gamma} d \log f(z)+\int_{\gamma} d \log c=\log \chi_{D}(\gamma),
$$

so we see the multiplier $\chi_{D}$ actually depends only on $D$ and not on the particular multiplicative meromorphic function chosen.

The preceding discussion shows that $\chi_{D}$ is a homomorphism of $H_{1}(V, Z)$ (the lower index denotes homology) into $C_{1}^{*}$, the multiplicative group of complex numbers of modulus 1 . Such a homomorphism is called a character of $H_{1}(V, Z)$, [12]. Let $Y$ be the direct product of the group $Z$ and the multiplicative group of positive real numbers. Let a be a positive real number so that ar is a representative of an element of $H_{1}(V, Y)$. Then

$$
\int_{\kappa_{\gamma}} d \log f(z)=\sigma \int_{\gamma} d \log f(z)=\alpha \log \chi_{D}(\gamma)=\log \left(\chi_{D}(\gamma)^{\alpha}\right),
$$

so

$$
\chi_{D}(\alpha \gamma)=\left[\chi_{D}(\gamma)\right]^{\alpha}
$$

Thus $\chi_{D}$ defines a character of $H_{1}(V, Y)$.

The character group (or dual group) of $Y$ is $C^{*}$, so a character of $H_{1}(V, Y)$ is an element of $H^{1}\left(V, C^{*}\right)$.

The isomorphism $\rho$ of $\left(11\right.$, ii) induces an isomorphism $\rho^{*}$ of $H^{1}(V, Z)$ into $H^{1}\left(V, C^{*}\right)$. As we have seen above, the character $\chi_{D}$ determines an element of $H^{1}\left(V, C^{*}\right)$ which we also denote by $\chi_{D}$. We say the character $\chi_{D}$ is $\rho$-trivial if it lies in the image of $H^{1}(V, Z)$ under $\rho^{*}$. The isomorphism $\rho$ also induces the homomorphism $\rho^{\prime}$ of $H^{1}(V, Z)$ into $H^{1}\left(V, \Omega^{*}\right)=\mathscr{F}$, shown in $(12, \mathrm{ii})$.

If we denote by $C^{*}$ the sheaf of non-vanishing constants over $V$ then $H^{1}\left(V, C^{*}\right)$ is isomorphic in a natural way to $H^{1}\left(V, C^{*}\right)$ and we may identify them. There is an inclusion map $i: C^{*} \subset \Omega^{*}$ and this induces a homomorphism $i^{*}$ of $H^{1}\left(V, C^{*}\right)$ into $H^{1}\left(V, \Omega^{*}\right)=\mathscr{F}$. Thus we have $0 \rightarrow H^{1}(V, Z) \stackrel{\rho^{*}}{\longrightarrow} H^{1}\left(V, C^{*}\right) \stackrel{i *}{\longrightarrow} \mathscr{F}$.

From this we see that $\rho^{\prime}=i^{*} \rho^{*}$.

Let $\sigma^{\prime}$ denote the homomorphism of $\mathscr{F}$ into $\mathscr{B}$ induced by the homomorphism $\sigma$ of $C^{*}$ into $T$. The images under $\sigma^{\prime}$ of elements of $H^{1}(V, Z)$ are all sent into the "zero" of $\mathscr{S}$ under $\sigma$. 'The following theorem is the result of this discussion.

THEOREM II. Two divisors $D^{\prime}$ and $D^{\prime \prime}$ on $V$ are $\omega$-equivalent if and only if

(i) $D^{\prime}$ is homologous to $D^{\prime \prime}$

(ii) The character of $D^{\prime}-D^{\prime \prime}$ is $\rho$-trivial. 
We should, of course, make explicit the observation that the isomorphism $\rho$ depends on $\sigma$ in its definition, and $\sigma$ depends on $\omega$. The isomorphism $\rho$ is not unique, but it is quite clear that our results are the same for any $\rho$ compatible with $\omega$. We can view this theorem as a sort of Abel's theorem for $\omega$-equivalence instead of linear equivalence [17].

We should further remark that, since any element of $H^{1}\left(V, C^{*}\right)$ is the image of an element of $H^{1}(V, Z)$ under the homomorphism $\rho^{*}$ induced by some isomorphism $\rho: Z \rightarrow C^{*}$, and since $\rho$ determines $\omega$ (because $\rho$ determines $\sigma$ by $C / \rho(Z)$, and $\omega=\sigma e)$ we see that two divisors are $\omega$ equivalent, for some $\omega$, if they are homologous. We state this formally as an addition to theorem II.

THEOREM IIA. If two divisors on $V$ are homologous, they are $\omega$ equivalent for some $\omega$.

This shows that the set of $\omega$-equivalences, in some sense, bridges the gap between linear equivalence and continuous equivalence in algebraic geometry.

There is another question of some interest which arises naturally at this point. We shall not dwell on it here, as the author intends to consider it in a subsequent investigation (and in another contex). However, it is interesting enough to deserve mention. Given $\omega$ and $\omega^{\prime}$, when does $\omega$-equivalence imply $\omega^{\prime}$-equivalence? Another way of approaching it: given $\omega$, find all $\omega^{\prime}$ such that $\omega$-equivalence implies $\omega^{\prime}$-equivalence. An obvious set of homomorphisms $\omega^{\prime}$ is available, but sometimes there are others. This is the question of the complex multiplications introduced into the theory of elliptic functions by Abel [1]. For a brief account see [9].

4. Hopf manifolds. H. Hopf has defined a class of compact complex manifolds each member of which is topologically equivalent to the product of a circle and an odd dimensional sphere, $S^{1} \times S^{-n+1}$, [8]. These manifolds may be generated as follows: let $E_{n+1}$ denote the space of the complex variables $\left(z_{0}, \cdots, z_{n}\right)$, and $E_{n+1}^{*}$ is $E_{n+1}$ with the origin $(0, \cdots, 0)$ deleted. Let $\mu$ denote the transformation on $E_{n+1}^{*}$ which sends $\left(z_{0}, \cdots, z_{n}\right)$ into $\left(2 z_{0}, \cdots, 2 z_{n}\right), \mu^{2}=\mu \cdot \mu$ sends $\left(z_{0}, \cdots, z_{n}\right)$ into $\left(4 z_{3}, \cdots, 4 z_{n}\right)$ etc. Let $\Delta$ denote the group generated by $\mu$. We define $\mathfrak{S}^{n+1}$ to be $E_{n+1}^{*} \bmod \Delta$.

Clearly $\mathfrak{Q}^{n+1}$ is topologically equivalent to $S^{1} \times S^{2 n+1}, \mathfrak{g}^{1}$ is just a torus, and $\mathfrak{g}^{n+1}$ carries a complex structure for $n=0,1,2, \ldots$. Furthermore, instead of choosing $J$ as a group generated by $2 \in C^{*}$ we could have used $\Delta_{\tau}$, generated by $\tau \in C^{*}$, satisfying $|\tau| \neq 1$, and obtained an $\mathfrak{g}^{n+1}$ which is also topologically $S^{1} \times S^{2 n+1}$, but has different analytic structures (in 
general) for different $\tau$. For the present we shall concern ourselves with $\mathfrak{S}^{n+1}=\mathfrak{S}_{2}^{n+1}$ only.

A compact Kähler manifold must have non-vanishing second Betti number: but $R_{2}=0$ for $\mathfrak{S}^{n+1}$ when $n>0$, thus $\mathfrak{S}^{n+1}$ is not Kähler and $a$ fortiori not algebraic, for $n>0$. This was (to the best of my knowledge) the first example given of a compact complex manifold which could not carry a Kähler structure. A closer study of this example will show us that, actually, the compact Kähler manifolds form a very small subclass of the compact complex manifolds.

First we must observe that $\mathfrak{g}^{n+1}$ is a $T$-bundle ${ }^{6}$ over the complex projective $n$-space $S_{n}$. Let $L$ denote a complex line through the origin of $E_{n+1}$, and let $L^{*}$ be that part of $L$ which is in $E_{n+1}^{*}$; then $\Delta$ maps $L^{*}$ into itself, and $L^{*} \bmod \Delta$ is isomorphically equivalent to $T$. An $L^{*}$ passes through each point in $E_{n+1}^{*}$, and no two $L^{*}$ 's intersect, hence $\mathfrak{g}^{n+1}$ is fibred by T's. The lines through the origin in $E_{n+1}$ constitute $S_{n}$, hence each $T \subset \mathfrak{S}^{n+1}$ corresponds in a unique way to a point of $S_{n}$. The mapping $\mathfrak{S}^{n+1} \rightarrow S_{n}$ defined by this correspondence is easily shown to be analytic.

Proposition 1. Every subvariety $V$ of $\mathfrak{g}^{n+1}$ is that part of $\mathfrak{g}^{n+1}$ which lies over a subvariety of $S_{n}$.

Proof. Let $\mathfrak{p}$ be a point of $V$, and let $T \mathfrak{p}$ be that $T \subset \mathfrak{g}^{n+1}$ which passes through $\mathfrak{p}$. We must show that $T_{\mathfrak{p}}$ lies entirely in $V$. Suppose not, then the intersection number $(V \circ T)>0$, for the local contribution to the intersection number is non-negative at each point and positive at least at $\mathfrak{p}$. But this is impossible since $T_{\mathfrak{p}} \sim 0$, hence $T_{\mathfrak{p}} \subset V$ and we thus conclude that $V$ is fibred.

Proposition 2. The group of divisors on $\mathfrak{S}^{n+1}$ is isomorphic in a natural way to the group of divisors on $S_{n}$.

In view of Proposition 1, we have only to remark that the coefficients of the components of the divisors are associated in the obvious manner. In fact Proposition 2 could have actually asserted an isomorphism between the group of algebraic 2k-cycles of $S^{n+1}$ and the group of algebraic $2(k-1)$-cycles of $S_{n}$.

Proposition 3. Every meromorphic function on $\mathfrak{W}^{n+1}$ is constant along the fibres and is thus associated in a bi-unique way with a rational function on $S_{n}$.

This also follows directly from Proposition 1, since each meromorphic

${ }_{6}$ We shall often refer to the bundle space as if it were the bundle and vice versa; of course, the bundle space by no means determines the bundle, but in each instance the exact meaning of the sentence is clear, and no ambiguity arises. 
function defines subvarieties. Thus the field of meromorphic functions over $\mathfrak{S}^{n+1}$ is the field of rational functions in $n$ variables.

COROLlaRy. The divisor class group on $\mathfrak{S}^{n+1}$ is isomorphic in a natural way to the divisor class group on $S_{n}$.

Let $Q$ be a point of a complex manifold, by a $p$-direction at $Q$ we mean a non-degenerate ccmplex $p$-vector at $Q$. We shall need the following lemma.

LEMMA 1. In an algebraic variety $V$, there exists, at a non-singular point $Q$, an algebraic subvariety in each p-direction at $Q$.

Froof. Let $z^{1}, \cdots, z^{n}$ be local coordinates at $Q$. Since $V$ is algebraic, and $Q$ is non-singular, there exist functions $w^{1}, \cdots, w^{n}$, rational on $V$, which form a new local coordinate system at $Q$. The equations $w^{p+1}=$ $\cdots=w^{m}=0$ define an algebraic subvariety of $V$ which passes through $Q$ in the $p$-direction of the $p$-vector $\mathfrak{b}_{p}$ determined by the coordinates $w^{1}, \cdots, w^{p}$. Let $\mathfrak{v}_{p}^{\prime}$ be a given $p$-vector at $Q$. There exists a linear transformation in the tangent space (of $V$ ) at $Q$ which sends $\mathfrak{v}_{p}$ into $\mathfrak{v}_{p}^{\prime}$. This sends $w^{1}, \cdots, w^{n}$ into $u^{1}, \cdots, u^{n}$, where the $u$ 's are linear combinations of the $w^{\prime}$ s with constant coefficients. The $p$-vector $\mathfrak{v}_{p}^{\prime}$ is determined by $u^{1}, \cdots, u^{p}$, and $u^{p+1}=\cdots=u^{n}=0$ defines an algebraic subvariety of $V$ passing through $Q$ in the $p$-direction $\mathfrak{b}_{p}^{\prime}$, which is what we wanted to prove.

We can now prove a theorem which indicates the value of examining the Hopf manifolds.

Theorem III. Let $V$ be any subvariety (of dimension greater than zero) of $S_{n}$ and let $B$ be the part of $\mathfrak{S}^{n+1}$ which lies over $V$, then $B$ is non-algebraic.

Proof. Since $V$ must have non-singular points, so must $B$. Let $Q$ be a non-singular point of $B$; choose local coordinates $z^{1}, \cdots, z^{n+1}$ at $Q$. so that the local equations of the fibre through $Q$ are $z^{1}=\cdots=z^{n}=0$. Choose any $p$-direction through $Q$ defined in terms of $z^{1}, \cdots, z^{n}$. If $B$ were algebraic there would exist a subvariety of $B$ passing through $Q$ in this $p$-direction. But this would be a subvariety of $\mathfrak{S}^{n+1}$ which did not contain the whole fibre passing through a point, $Q$, of it. By Proposition 1, such subvarieties do not exist, hence $B$ is not algebraic. Let us note explicitly that we did not require either $V$ or $B$ to be nonsingular. 
To distinguish $\mathfrak{X}^{n+1}$ explicitly (from among all other $T$-bundles over $S_{n}$ ) we should give its coordinate transformations. We have said that $S_{n}$ consists of the set of (complex) lines through the origin in $E_{n+1}$; let us complete $E_{n+1}$ into $S_{n+1}$ by adding $\tilde{S_{n}}$, a projective $n$-space "at infinily", to $E_{n+1}$. Let $O$ be the point of $S_{n+1}$ which comes from the origin in $E_{n+1}$, and we replace $O$ by $S_{n}$, that is, we perform a (local) quadratic transformation (Hopf star-process) at $O$, this gives rise to a new space $S_{n+1}^{*}$ (not a projective space). $S_{n+1}^{k}$ is a line bundle, in the sense of Kodaira and Spencer, over $S_{n}$. Let $S_{n}^{\prime}$ be an $n$-dimensional linear subspace of $S_{n+1}$ which does not pass through $O$. Let $\widetilde{S}_{n-1}=\left(S_{n}^{\prime} \circ \widetilde{S}_{n}\right)$ be the linear subvariety of $\tilde{S}_{n}$ which is the intersection. A unique subvariety $S_{n-1}$ of $S_{n}$ corresponds to $\tilde{S}_{n-1}$ in the obvious way (by bundle projection on $S_{n}$ ). Let us define the divisor $D$ on $S_{n}$ by $D=-S_{n-1}$. Since $S_{n-1}$ is a linear subspace of $S_{n}$ and sinze $z_{\lrcorner}, \cdots, z_{n}$, the coordinates in $E_{n+1}$, serve as homogeous coordinates in $S_{n}$, local minimal equations of $D$ are easily obtained and a $C^{*}$-bundle is defined, as described in $\S 1$. Let $f_{i j}(z)$ designate coordinate transformations of this $C^{*}$-bundle, where $z \in U_{i} \cap$ $U_{j} \subset S_{n}$, and let $\sigma: C^{*} \rightarrow T$ be defined by $C^{*} / \Delta=T$. Then $\theta_{i j}(z)=\sigma f_{i j}(z)$ are the coordinate transformations of $\mathfrak{g}^{n+1}$.

To proceed in another way, let $p: S^{: n+1} \rightarrow S_{n}$ be the Hopf map, i.e. the point $\left(z_{3}, \cdots, z_{n}\right) \in S^{n+1}$, where $\left|z_{3}\right|^{2}+\cdots+\left|z_{n}\right|^{2}=1$, goes into $\left(z_{0}, \cdots\right.$, $\left.z_{n}\right) \in S_{n}$, where $z_{j}, \cdots, z_{n}$ are homogeneous coordinates on $S_{n}$ [7]. This defines $S^{2 n+1}$ as a bundle of circles over $S_{n}$; denote the fibre by $\overline{S^{1}}$. Let $x \in S^{1}, \bar{s} \in S^{i n+1}$, and $q(x, \bar{s})=\bar{s}$, this defines a mapping of $S^{1} \times S^{i n+1}$ onto $S^{i n+1}$. Let $T=S^{1} \times \overline{S^{1}}$. We consider the following arrangement

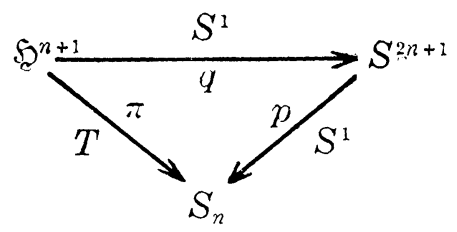

If $t_{i j}(z)$ are the coordinate transformations of $S^{2 n+1}$ over $S_{n}$, then $\theta_{i j}(z): T \rightarrow T$, the coordinate transformations of $\mathfrak{S}^{n+1}$ over $S_{n}$ are defined by

$$
\theta_{i j}(z):(x, \bar{x}) \rightarrow\left(x, t_{i j}(z) \cdot \bar{x}\right), \quad z \in U_{i} \cap U_{j}, x \in S^{1}, \bar{x} \in \bar{S}^{1} .
$$

If we should replace $T$ as fibre by any other complex manifold $F$, on which $T$ may act as transformation group through the coordinate transformations $\theta_{i j}$, we get a $T$-bundle with fibre $F$, and the bundle space of this bundle is a complex manifold. Now let $S^{1}$ be contained in $S^{2 m+1}$ as one of the Hopf fibres over $S_{m}$. Then

$$
T=S^{1} \times{\overline{S^{1}}} \overline{\mathfrak{S}}^{m+1}=S^{m+1} \times{\overline{S^{1}}}^{1}
$$


Since the transformations $\theta_{i j}$ all act on $T$ in such a way that $S^{1}$ is left fixed, we see that $\theta_{i j}: \overline{\mathfrak{S}}^{m+1} \rightarrow \overline{\mathfrak{S}}^{m+1}$. Thus we get a bundle $M_{m, n}$ over $S_{n}$ with the Hopf manifold ${\overline{\mathfrak{S}^{m}}}^{m}$ as fibre.

Proposition 4. The bundle $M_{m, n}$ is topologically equivalent to $S^{2 m+1} \times S^{2 n+1}$.

Proof. Let $\pi^{\prime}$ be the projection of $M_{m, n}$ onto $S_{n}$ (induced by the $\pi$ of $\left.\$_{2}^{n+1}\right)$. Let $P$ be a point of $M_{m, n}$ and $z=\pi^{\prime}(P)$. The point $P$ is represented by a system of pairs $\left(h_{i}, z\right)$, where $z \in U_{i}$ and $h_{i} \in{\overline{\mathfrak{S}^{2}}}^{m+1}$; if $z \in U_{j}$ also, then the pair $\left(h_{j}, z\right)$ represents the same point if $\theta_{i j}(z) \cdot h_{j}=h_{i}$. Since ${\overline{\mathfrak{S}^{m+1}}}^{m}=S^{2 m+1} \times{\overline{S^{1}}}^{1}, h_{i}=\left(s_{i}, \bar{x}_{i}\right)$ where $s_{i} \in S^{2 m+1}$ and $\bar{x}_{i} \in \bar{S}^{1}$. But $\theta_{i j}$ does not act on $S^{2 m+1}$ (perhaps we should say its action is the identity), so we may drop the subscript on $s_{i}$ and write $h_{i}=\left(s, \bar{x}_{i}\right)$. Thus the point $P$ is represented by $\left(s, \bar{x}_{i}, z\right)$. If we note furthermore that

$$
\theta_{i j}(z) \cdot h_{j}=\theta_{i j}(z)\left(s, \bar{x}_{j}\right)=\left(s, \bar{x}_{i}\right)=\left(s, t_{i j}(z) \cdot \bar{x}_{j}\right),
$$

we see that the pair $\left(\bar{x}_{i}, z\right)$ represents a point $\bar{s}$ of $S^{2 n+1}$. Consequently $P$ is represented by the pair $(s, \bar{s})$. This shows that $M_{m, n}$ is topologically equivalent to $S^{2 m+1} \times S^{2 n+1}$.

The manifolds $M_{m, n}$ were recently introduced and studied, from a somewhat different point of view, by E. Calabi and B. Eckmann [2]. In fact, Calabi and Eckmann view $M_{m, n}$ as a $T$-bundle over $S_{m} \times S_{n}$.

Let $r=(m+1)(n+1)-1$, let $w_{0}, \cdots, w_{m}$ be homogeneous coordinates in $S_{m}$, let $z_{0}, \cdots, z_{n}$ be homogeneous coordinates in $S_{n}$, and let $\zeta_{i j}, i=0$, $\cdots, m ; j=0, \cdots, n$, be homogeneous coordinates in $S_{r}$. We may define a natural imbedding of $S_{m} \times S_{n}$ into $S_{r}$ by setting $\zeta_{i j}=w_{j} \cdot z_{j}, i=0, \cdots, m$, $j=0, \cdots, n$. Let $k>r$, and let $S_{r}$ be a linear subspace of $S_{k}$; we say that $S_{m} \times S_{n}$ has the natural imbedding in $S_{k}$ if we imbed it naturally in $S_{r}$ and then consider it a subspace of $S_{k}$, [6].

Proposition 5. Let $k \geqq(m+1)(n+1)-1$, and let $S_{m} \times S_{n}$ have the natural imbedding in $S_{k}$, then the part of $\mathfrak{g}^{k+1}$ which lies over $S_{m} \times S_{n}$ is the manifold $M_{m, n}$.

This follows directly from the discussion centering around Proposition 4.

Remark. The part of $\mathfrak{S}^{k+1}$ which lies over $S_{r} \subset S_{k}$ is $\mathfrak{g}^{r+1}$.

The Calabi-Eckmann manifolds $M_{m, n}$, excluding the Hopf manifolds $M_{1, n}=\mathfrak{S}^{n+1}$, occur as special cases of a class of manifolds studied by $\mathrm{H}$. 
C. Wang [16]. By a Wang manifold we mean a compact complexhomogeneous space which is simply connected. (Actually it is only necessary to assume that the fundamental group is finite, but this causes statements and proofs to be cumbersome, and we can expedite matters by assuming simple connectedness; the necessary modifications to prove the more complete statements will be obvious in every instance.) On the basis of Wang's work, M. Goto has shown:

"A Wang manifold is a complex fibre bundle, with a torus as the fibre, over a rational variety," [5].

We should remark that, as used here, "torus" means a complex torus of arbitrary complex dimension including zero. If the torus is of dimension zero, this means the Wang manifold is itself a rational variety.

Theorem IV. A Wang manifold $W$ is Kählerian if and only if its Euler characteristic $\chi(W)$ is non-vanishing. If it is Kählerian, it is algebraic, and even rational.

Before we can prove the theorem, we need a simple lemma.

Lemma 2. If $F$ is a subvariety of $V$, and if $F$ is homologous to zero on $V$, then $V$ is non-Kählerian.

Proof. Suppose the complex dimension of $F$ is $m$, and of $V$ is $n$, and suppose $V$ is Kählerian. Let $\omega$ be the Hodge form on $V$, that is, the exterior 2-form associated with the Kähler metric, and let $\omega^{m}$ be the exterior product of $\omega$ with itself $m$ times. Then

$$
\int_{F} \omega^{m} \neq 0 .
$$

(This integral is not zero since $\omega^{m}$ is a volume element on $F$ with the induced metric.) But this integral is essentially the Kronecker index of the cocycle $\omega^{m}$ and the cycle $F$; since it does not vanish, $F$ cannot be homologous to zero. This lemma is merely an old theorem of Lefschetz', on algebraic varieties, stated backwards. The Kählerian dressing is, in view of Hodge's work, a trivial addition.

Proof of theorem $I V$. If $W$ is fibred by tori then $\chi(W)=0$. Thus, if $\chi(W) \neq 0$, we see by Goto's theorem that $W$ is a rational manifold. Now suppose $\chi(W)=0$ and let $T$ be a fibre. $T$ is itself a group, and can be considered as a subgroup of the group, say $G$, acting on $W$. Let the complex dimension of $T$ be $m$ and let $C_{1}, \cdots, C_{2 m}$ be closed (real) one parameter subgroups of $T$ whose point sets are the carriers of basic 1-cycles of $T$. Let $E$ be the closed subgroup of $T$ generated by $C_{1}, \cdots$, $C_{2 m-1}$; it is the carrier of a basic $(2 m-1)$-cycle on $T$. Let $x \in E$ and 
$C_{x}$ be the 1-cycle on $T$ into which $x$ sends $C_{2 m}$, that is, the coset of $x \cdot C_{2 m}$ of $C_{2 m}$. W is simply connected, so there exists a 2-chain (actually a 2-cell) $\Gamma^{2}$ such that $C_{2 m}$ bounds $\Gamma^{2}$. Since we can view $x$ as an element of $G$, it sends $\Gamma^{2}$ onto some 2 -chain $\Gamma_{x}^{2}$ such that $C_{x}$ bounds $\Gamma_{x}^{2}$. As $x$ ranges over $E$, the $\Gamma_{x}^{2}$ sweeps out a $(2 m+1)$-chain $\Gamma^{2 m+1}$. Now $T$ bounds $\Gamma^{2 m+1}$, so is homologous to zero in $W$; by the lemma this shows that $W$ is non-Kählerian. (We remark that all chains and cycles to which we refered are singular chains and cycles.)

The Hopf manifolds can be utilized in still another construction which proves interesting. Let $E_{n+1}, E_{n+1}^{*}, \mu, \Delta$, etc., be as before; let $L_{k+1}$ denote a linear (complex) $(k+1)$-space through the origin of $E_{n+1}$ and $L_{k+1}^{*}$ the part of $L_{k+1}$ in $E_{n+1}^{*}$. Each $L_{k+1} \subset E_{n+1}$ is a point of the complex Grassmann variety $\Omega(k, N)$. The group $\Delta$ maps $L_{k+1}^{*}$ into itself, so each $L_{k+1}$ corresponds uniquely to an $\mathfrak{S}^{k+1} \subset \mathfrak{2}^{n+1}$, consequently $\Omega(k, N)$ can be looked upon as a set of $\mathfrak{S}^{k+1}$ s in $S_{2}^{n+1}$. This gives a method for generating $\mathfrak{S}^{k+1}$ bundles over an arbitrary compact complex variety $V$. (By a complex variety, we mean a subvariety, not necessarily non singular, of some complex manifold.)

Let $\varphi$ be an analytic mapping of $V$ into $\Omega(k, N)$; we may generate, in the usual manner, an $\mathfrak{S}^{k+1}$ bundle whose bundle space we may denote by $B(V, k, N, \varphi)$. Actually, for fixed $V, k, N, \varphi$, we get a continuum of analytically distinct $B(V, k, N, \varphi)$ by varying the analytic structure of $T$, thus of $\mathfrak{S}^{k+1}$.

\section{Proposition 6. Evevy $B(V, k, N, \varphi)$ is non-Kählerian.}

This is obvious, since a non-singular subvariety of a Kähler variety must be Kähler; but the fibres of each $B(V, k, N, \varphi)$ are non-Kählerian. This shows that the Kähler varieties must be a very small subclass of the class of compact complex manifolds. In particular, to each algebraic variety there corresponds, in a natural way, a continuum of distinct, non-trivial, non-Kählerian varieties.

Proposition 7. The Calabi-Eckmann varieties are all generated by linear varieties which lie on Grassmann varieties.

This follows directly from the details of the construction described above and the discussion concerning Proposition 4.

Our construction can be generalized further. Let $K$ be any complex bundle of (complex) linear spaces over $V$, and let $L_{r+1}$ be the fibre of the bundle, [15]. We may reduce $L_{r+1}^{*}$ by $\Delta$ to get $K^{*}$, an $\mathfrak{S}^{r+1}$ bundle 
over $V$. This construction has another interesting aspect. Let $M$ be the bundle space of the complex sphere bundle arising from $K$, [3]. Let $S^{2 r+1}$ be the fibre of the bundle $M$, and let $S^{1}$ be such that $S^{2 r+1} \times S^{1}=\mathfrak{S}^{r+1}$.

Proposition 8. If $M$ is the bundle space of a complex sphere bundle over $V$ which arises from a complex bundle of linear spaces then $M \times S^{1}$ carries natural complex structures all of which are non-Kählerian.

Since the bundle group does not act on $S^{1}$, in the product $S^{2 r+1} \times S^{1}$, we see that $M \times S^{1}$ is an $\mathfrak{S}^{r+1}$ bundle over $V$. In the statement that all are non-Kählerian, we of course understand that $r>0$.

We cannot resist mentioning that the construction can be pushed still further, since all we really need from $K$, the bundle of linear spaces, is the underlying projective bundle. As one knows, not every projective bundle arises as the underlying projective bundle of a bundle of linear spaces, so this gives us a yet larger construction. Of course, the path is now clear to begin new constructions by considering bundles with some of these new non-Kählerian manifolds we have constructed as fibres and so on ad infinitum.

\section{REFERENCES}

1. N. H. Abel, Theoremes et problems, J. für Math., 2 (1827), 286, also see, Recherches sur les fonctions elliptiques, Oeuvres Complètes, Tome I, Christiania, 1839, pp. 141-252.

2. E. Calabi and B. Eckmann, A class of compact complex manifolds which are not algebraic, Ann. Math., 58 (1953), 494-500.

3. S. S. Chern, Characteristic classes of Hermitian manifolds, Ann. Math., 47 (1946), 85-121.

4. P. R. Garabedian and D. C. Spencer, A complex tensor calculus for Kähler manifolds, Acta Math., 89 (1953), 279-331.

5. M. Goto, On algebraic homogeneous spaces, Amer. J. Math., 76 (1954), 811-818.

6. W. V. D. Hodge and D. Pedoe, Methods of algebraic geometry, Cambridge University Press, 1952.

7. H. Hopf, Über die Abbildungen von Spharen auf Spharen niedrigerer Dimension, Fund. Math., 25 (1935), 427-40.

8. …-, Zur Topologie der komplexen Mannigfaltigkeiten, Courant Anniversary Volume, New York, 1948, pp. 167-185.

9. C. Jordan, Cours d'analyse, Tome 2, Paris, 1894. (In particular pp. 531-538.)

10. K. Kodaira and D. C. Spencer, Groups of complex line bundles over compact Kähler varieties, and divisor class groups on algebraic varieties, Proc. Nat. Acad. Sci. U.S.A., 39 (1953), 868-77.

11. S. Lefschetz, L'analysis situs et la geometrie algebrique, Paris, 1924.

12. _ _ Algebraic topology, Amer. Math. Soc. Coll. Publ. Vol. XXVII, 1942.

13. G. de Rham and K. Kodaira, Harmonic integrals, Institute for Advanced Study notes, 1950.

14. D. C. Spencer, Sheaves, Princeton University notes, 1954. 
15. N. E. Steenrod, Topology of fibre bundles, Princeton University Press, 1951.

16. H. C. Wang, Closed manifolds with homogeneous complex structure, Amer. J. Math., 76 (1954), 1-32.

17. H. Weyl, Die Idee der Riemannschen Fläche, Leipzig, 1923 and New York, 1947.

18. O. Zariski, Algebraic surfaces, Ergebnisse der Mathematik und ihrer Grenzgebiete, Vol. 3 (1935).

UNIVERSITY OF ILLINOIS 


\section{PACIFIC JOURNAL OF MATHEMATICS}

\section{EDITORS}

H. L. Royden

Stanford University

Stanford, California

E. HewitT

University of Washington

Seattle 5 , Washington
R. P. Dilworth

California Institute of Technology Pasadena 4, California

E. G. Straus

University of California

Los Angeles 24, California

\section{ASSOCIATE EDITORS}

E. F. BECKENBACH

C. E. BURGESS

H. BUSEMANN

H. FEDERER

\author{
M. HALL \\ P. R. HALMOS \\ V. GANAPATHY IYER \\ R. D. JAMES
}

M. S. KNEBELMAN

I. NIVEN

T. G. OSTROM

M. M. SCHIFFER
J. J. STOKER

G. SZEKERES

F. WOLF

K. YOSIDA

\section{SUPPORTING INSTITUTIONS}

UNIVERSITY OF BRITISH COLUMBIA

CALIFORNIA INSTITUTE OF TECHNOLOGY

UNIVERSITY OF CALIFORNIA

MONTANA STATE UNIVERSITY

UNIVERSITY OF NEVADA

OREGON STATE COLLEGE

UNIVERSITY OF OREGON

UNIVERSITY OF SOUTHERN CALIFORNIA
STANFORD UNIVERSITY

UNIVERSITY OF UTAH

WASHINGTON STATE COLLEGE

UNIVERSITY OF WASHINGTON

AMERICAN MATHEMATICAL SOCIETY CALIFORNIA RESEARCH CORPORATION HUGHES AIRCRAFT COMPANY 


\section{Pacific Journal of Mathematics}

\section{Vol. 6, No. $1 \quad$ November, 1956}

David Blackwell, An analog of the minimax theorem for vector payoffs..... 1

L. W. Cohen, A non-archimedian measure in the space of real

sequences ..................................... 9

George Bernard Dantzig, Constructive proof of the Min-Max theorem ..... 25

Jim Douglas, On the numerical integration of quasilinear parabolic

differential equations ............................... 35

James Michael Gardner Fell, A note on abstract measure ............. 43

Isidore Isaac Hirschman, Jr., A note on orthogonal systems . . . . . . . . . . 47

Frank Harary, On the number of dissimilar line-subgraphs of a given

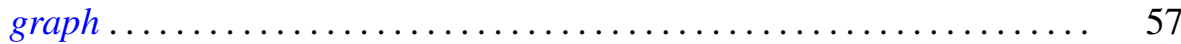

Newton Seymour Hawley, Complex bundles with Abelian group......... 65

Alan Jerome Hoffman, Morris Newman, Ernst Gabor Straus and Olga

Taussky, On the number of absolute points of a correlation ...........

Ernst Gabor Straus and Olga Taussky, Remark on the preceding paper.

Algebraic equations satisfied by roots of natural numbers . . ........ 97

Ralph D. James, Summable trigonometric series ................. 99

Gerald R. Mac Lane, Limits of rational functions . . . . . . . . . . . . . . . 111

F. Oberhettinger, Note on the Lerch zeta function ................. 117

Gerald C. Preston, On locally compact totally disconnected Abelian groups and their character groups ........................... 121

Vikramaditya Singh and W. J. Thron, On the number of singular points, located on the unit circle, of certain functions represented by

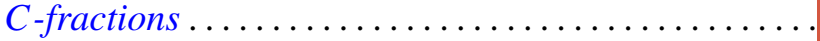

Sherman K. Stein, The symmetry function in a convex body ... 145 Edwin Weiss, Boundedness in topological rings.............

Albert Leon Whiteman, A sum connected with the series for the partition

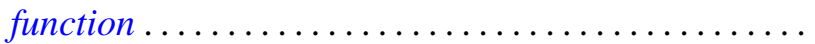

Alfred B. Willcox, Some structure theorems for a class of Banach algebras.

Joseph Lawrence Zemmer, Some remarks on p-rings and their Boolean geometry... 